\title{
Intestinal permeability in rats infected by Nippostrongylus brasiliensis
}

\author{
I. COBDEN, J. ROTHWELL, AND A. T. R. AXON \\ Fiom the Gastroenterology Unit, The General Infirmary, Leeds
}

SUMmaRY Passive intestinal permeability has been investigated in rats infected by the nematode, Nippostrongylus brasiliensis, by the simultaneous administration of two probe molecules. Experiments using a closed intestinal loop show that there is a significantly increased absorption of lactulose and decreased absorption of mannitol in rats at the 10th to 11th day of infection. Experiments using serial oral administration techniques show that these changes start during the second week of the infection with a return towards normal values by the end of the third week. The results are similar to those found in human coeliac disease and add weight to previous studies which have stressed the similarity in the two disease processes.

Nippostrongylus brasiliensis is a nematode which infects rats, predominantly colonising the proximal small intestine. In addition to providing a convenient animal model for the study of hookworm infestations, the immunological mechanisms involved in recovery from infection and the associated intestinal lesion have both frequently been investigated. The larvae can be administered by subcutaneous injection, after which they begin to reach the duodenum and jejunum after three to four days (Symons and Fairbairn, 1960). As the worm does not multiply within the host, the degree of intestinal colonisation reflects the administered dose (Jenkins, 1974). The worms mature in the small intestine, feeding in close association to the intestinal mucosa and by the seventh or eighth day maximal egg production is occurring. During the second and third weeks the animal mounts an immune response and the infection is steadily thrown off.

Associated with the infection there is a patchy intestinal damage which is maximal at the 10th to 11 th day (Symons and Fairbairn, 1960; Ferguson and Jarret, 1975) resolving by the end of the third week. The lesion bears a striking similarity to that found in human coeliac disease, with villous atrophy, crypt hyperplasia, and changes in epithelial cells (Symons, 1965). This similarity extends to functional aspects. Symons has described decreased activity of brush border enzymes, and has also demonstrated impaired absorption of glucose and other nutrients (Symons and Fairbairn, 1963; Symons et al., 1971). As in coeliac disease (Croft et al., 1968), there is evidence of increased cell turnover (Symons, 1965;

Received for publication 21 February 1979
Loehry and Creamer, 1969). Moreover, Ferguson and Jarrett (1975), studying the pattern of infection in thymectomised rats, have suggested that the intestinal lesion is the result of a cell-mediated immune response by the host, rather than a direct toxic effect of the parasite.

We have previously described a non-invasive test of passive intestinal permeability based on the oral administration of two water-soluble molecules, one of which is absorbed to an increased, and the other to a decreased, extent in coeliac disease (Cobden et al., 1978a, b). The five hour recoveries of the two probe molecules, mannitol and cellobiose, were calculated and, when the result was expressed as a ratio, a clear separation was achieved between untreated adult coeliacs and controls.

Using a modification of this test, we have studied the changes in passive permeability occurring in rats infected with $N$. brasiliensis.

\section{Methods}

Two sets of experiments were carried out, the first involving the use of a closed intestinal segment, and the second using a gavage technique for administering the solution to conscious rats.

As in our human studies, we have expressed absorption in both experiments in terms of the quantities recovered in timed urinary collections. In the closed loop experiments, we could have assayed luminal concentrations instead, and thus indirectly calculated the quantities absorbed, but when studying a poorly absorbed molecule such as lactulose the method is almost certain to be too insensitive to detect even relatively large changes in absorption. 
Serial blood-sampling would be an alternative but would not measure total absorption. Although the influence of renal excretory function is a theoretical disadvantage of urinary collection as opposed to plasma sampling, it has the important practical consideration of concentrating the substances to be assayed to a high degree. Our present assay techniques relate to urinary concentrations, and we have found them to be both accurate and precise

For the gavage experiments, the use of timed urine collections has the added advantage of being completely non-invasive, enabling comparisons to be made between results for the same animal when studied repeatedly. It also allows us to draw comparisons with our human results.

\section{EXPERIMENT A (closed loop)}

The method used in this experiment was similar to that used by Symons et al. (1971), but, in addition, we had to incorporate a means for collecting urine.

Five male Wistar rats, weighing $150-200 \mathrm{~g}$, were each injected subcutaneously with 6000 larvae of $N$. brasiliensis, and the subsequent experiments performed at the time when the intestinal lesion could be expected to be at its height (three on the 10th and two on the 11th day after infection). After overnight fasting the animals were anaesthetised with intraperitoneal pentobarbitone $(30-60 \mathrm{mg} / \mathrm{kg})$. A low midline abdominal incision was made and the bladder identified. A loose ligature was passed around the bladder taking care to avoid the ureters. Through a small incision in the relatively avascular fundus, a plastic cannula with a flanged end was inserted, and the ligature tightened to retain the cannula by the flange and ensure that there was no leakage of urine around the cannula. The urethra was then ligated, and when urine was seen to be flowing down the cannula, the next stage of the experiment was started. A $20 \mathrm{~cm}$ segment of jejunum, beginning $5 \mathrm{~cm}$ distal to duodenojejunal flexure, was closed with ligatures, care being taken not to interfere with its blood supply and to avoid excessive trauma, which might result in renal shutdown. Two millilitres of the test solution were injected into the lumen through a fine-gauge needle, and the bowel returned to the abdominal cavity.

The test solution contained $5 \mathrm{~g}$ lactulose and $2 \mathrm{~g}$ mannitol dissolved in $100 \mathrm{ml}$ water $(100 \mathrm{mg}$ and 40 $\mathrm{mg}$ respectively in the $2 \mathrm{ml}$ dose). Although in our human experiments we have used hypertonic ( 1500 mosmol) solutions to enhance the absorption of disaccharide, this was not feasible in a closed loop because of the excessive distension that would result. The solution was therefore made up without any other osmotic filler to be more nearly isotonic (about 380 mosmol).
Urine was collected over the next two hours. At the end of this time, all urine was aspirated through the cannula, and, to ensure complete recovery from any dead-space, the bladder was irrigated with $0.5 \mathrm{ml}$ $0.9 \%$ saline, the resulting aspirate being added to the urine collection. The animal was killed, the closed intestinal loop removed, and the length measured avoiding excessive stretching (range from $17-22 \mathrm{~cm}$ ).

Specimens for histology were taken at the end of each experiment from the jejunum proximal to the closed segment, and from the segment itself. In addition, in two experiments specimens were taken from the proximal jejunum, at the time of ligating the closed loop at the beginning of the experiment. In practice, there were no significant histological differences between any of the specimens, allowing for the patchy nature of the intestinal lesion. Similar experiments were carried out on a control group of five uninfected rats.

\section{EXPERIMENT B}

Seven male wistar rats, weight $150-200 \mathrm{~g}$, were injected subcutaneously with 6000 larvae of $N$. brasiliensis. On the third day of the infection, after overnight fasting, the rats were gavaged with $1 \mathrm{ml}$ of a solution which was similar to that used in the previous experiment, except that it contained $21 \mathrm{~g}$ dextrose per $100 \mathrm{ml}$, making it strongly hypertonic (about $1500 \mathrm{~m} 0 \mathrm{smol}$ ). The animals were then placed in metabolism cages (Forth-Tech Ltd.) designed to collect urine and faeces separately, and urine was collected over eight hours. This was repeated on the seventh, 10th, 13th, and 21st days of the infection. Six uninfected rats, acting as a control group, were treated similarly on the corresponding days.

\section{ASSAY TECHNIQUES}

In each case, the volume of urine collected was measured. Samples were assayed for mannitol content by a modification of the spectrophotometric method of Corcoran and Page (1947), and for lactulose by quantitative paper chromatography (Menzies, 1973). The results for the closed loop experiments were expressed as the quantity recovered per $\mathrm{cm}$ of bowel (per two hour collection period). For the gavage experiments, results were expressed as the percentage of the administered oral dose recovered in the urine after eight hours. In both cases, the ratio of lactulose recovery $(\mathrm{mg}) /$ mannitol recovery $(\mathrm{mg})$ was calculated.

\section{Results}

HISTOLOGY

The typical changes induced by the parasite in the 

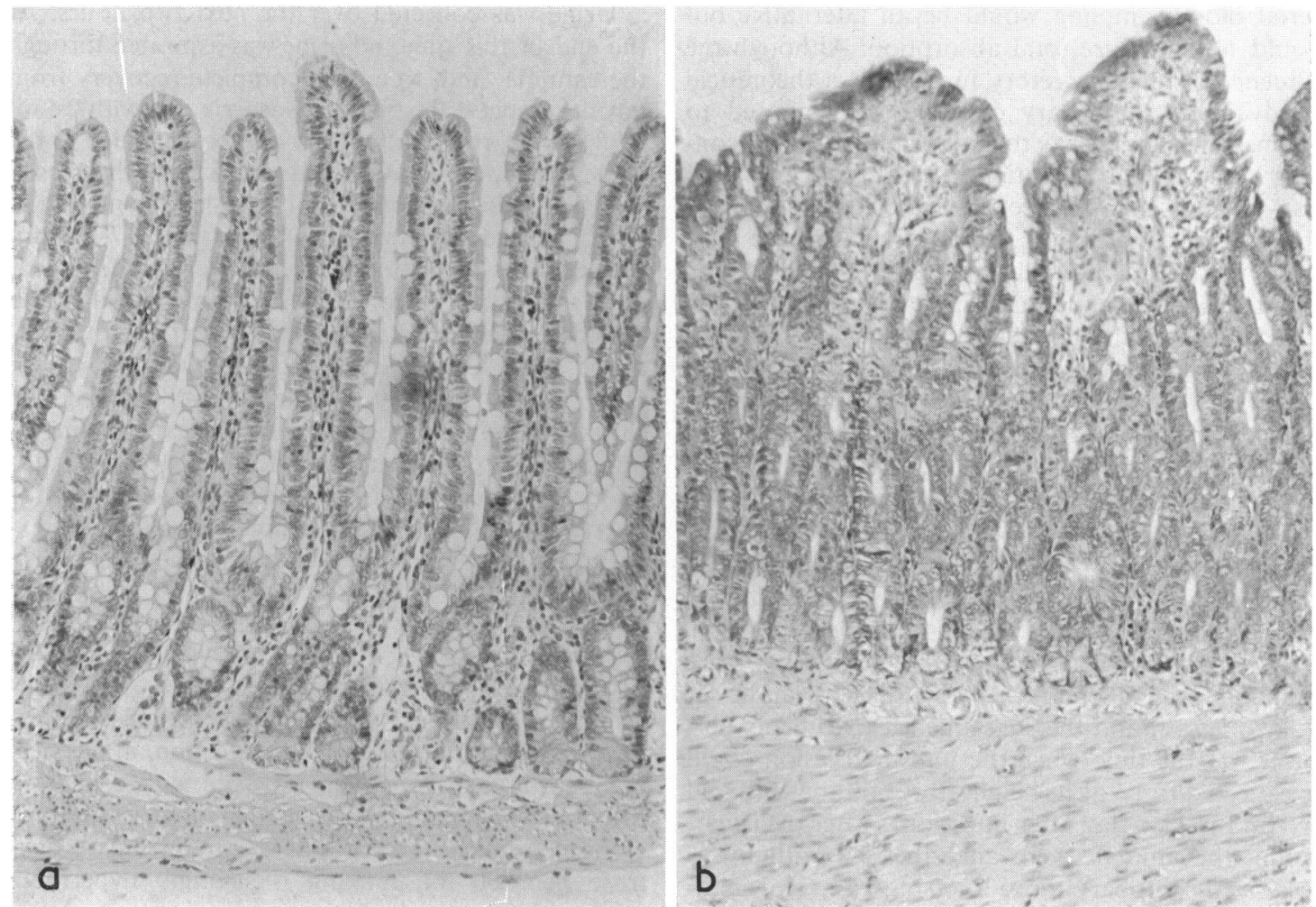

Fig. 1 Jejunal histology of control (a) and infected rats $(b) . \times 140$.

jejunum are shown in Fig. 1 with a sample from a control rat for comparison. There was a striking villous shortening, with crypt hyperplasia, epithelial cell changes, and an inflammatory infiltrate. The similarity to human coeliac disease is readily apparent.

\section{CLOSED LOOP EXPERIMENTS}

The results are shown in Fig. 2. There was a significant decrease in mannitol recovery in the infected rats compared with controls $(\mathrm{P}<0.005$, Student's $t$ test for small samples). In contrast, lactulose recovery was significantly increased $(\mathrm{P}<0.05)$. As a consequence, the ratios lactulose/mannitol were significantly higher in the infected rats $(\mathrm{P}<0.005)$.

\section{GAVAGE EXPERIMENTS}

The recovery of mannitol in the seven rats through the course of the infection is shown in Fig. 3. There was a significant decrease $(\mathrm{P}<0.005)$ on the 10th and 13th days of the infection when compared with the values on the third day (at which time the parasites would not be established in the intestine). By day 21, the mannitol recovery had risen again, although it was still less than that on day $3(\mathrm{P}<0.05)$.
In contrast, the lactulose excretion increased during the infection (Fig. 4) and fell again by day 21, although the rise just failed to reach statistical significance $(0 \cdot 1>P>0.05$, day 13$)$.

As a result, the ratio was significantly higher (Fig. 5 ) on days 10 and 13 , than on day $3(P<0.005)$, with little overlap. There was no significant difference on days 7 and 21, although one rat on day 7 had a high ratio.

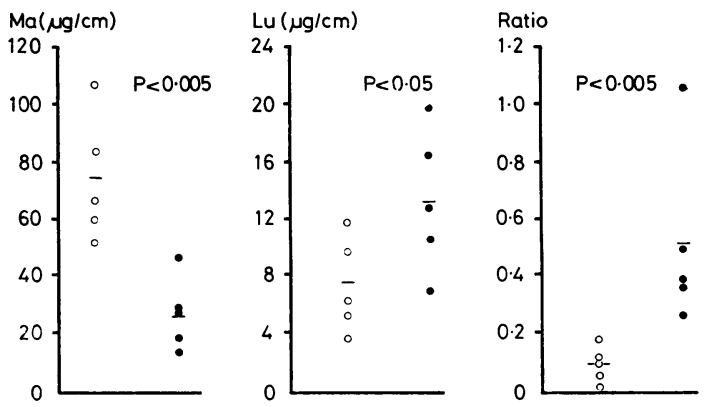

Fig. 2 Closed loop experiments: mannitol recovery, lactulose recovery and lactulose/mannitol ratios in two hour urine samples. Control. Infected. 


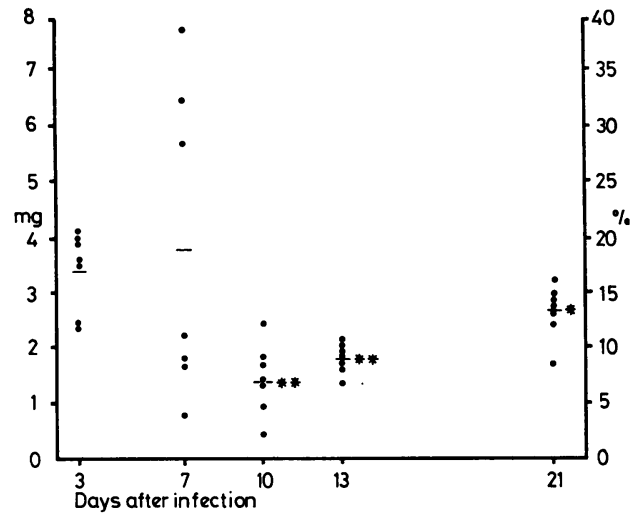

Fig. 3 Gavage experiments: mannitol recoveries in eight hour urine collections in infected rats

${ }^{*} \mathrm{P}<0.05,{ }^{* *} \mathrm{P}<0.005$ with respect to day 3 .

The control group of rats, subjected to the same gavagings, showed little fluctuation in the ratios throughout the experiment (Fig. 6-same scale).

\section{Discussion}

The results show that rats infected with $N$. brasiliensis undergo a transient change in passive intestinal permeability at about the time of the maximum histological damage. It is uncertain whether the decreased mannitol recovery on day 21 truly reflects residual functional impairment, in that the ratio lactulose/mannitol was unaltered. It could be argued that the effect was due to some non-mucosal factor -for example, more rapid intestinal transitwhich resulted in the absorption of both molecules being decreased. This is one of the advantages to be gained by the simultaneous administration of two molecules with contrasting changes in absorption in

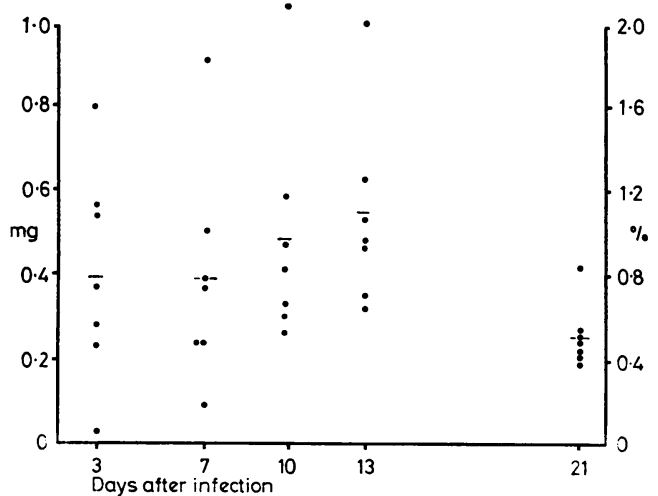

Fig. 4 Gavage experiments: lactulose recoveries in eight hour urine collections in infected rats.

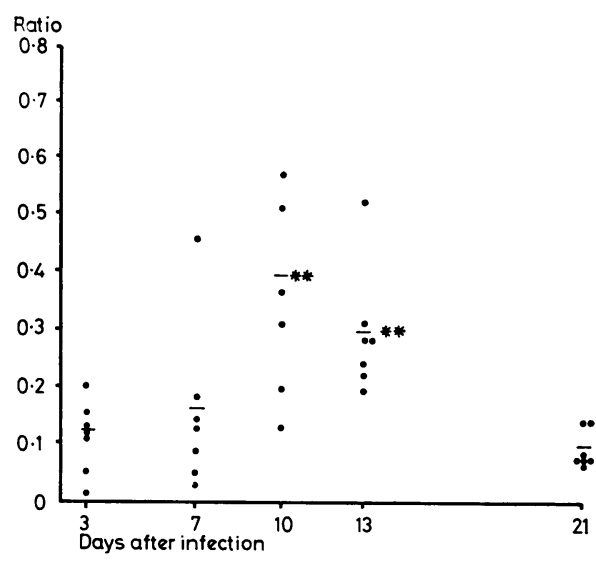

Fig. 5 Gavage experiments: lactulose/mannitol ratios in eight hour urine collections in infected rats ** $\mathrm{P}<0.005$ with respect to day 3 .

disease states; errors due to non-mucosal factors are minimised when the result is expressed as a ratio. The closed-loop experiment confirms that the increase in ratio on day 10 is a genuine reflection of altered permeability, as factors such as gastric emptying or intestinal transit rate obviously do not apply.

The probe molecules were chosen to straddle the effective pore size of the intestinal mucosa. Mannitol is a polyhydric alcohol of molecular radius c. $4 \AA$, similar in size to xylose, but, unlike xylose, having no affinity for active transport systems (Alvarado, 1966). Although it is frequently used as a nonabsorbable marker, our results both in humans (Cobden et al., 1978a, b) and in this experiment, and the work of others (Nasrallah and Iber, 1968), show that significant quantities are absorbed. This is not surprising, when one considers that a figure of $4 \AA$ has been calculated for the radius of the water-filled pores presumed to perforate the lipid-barrier of the small intestine (Lindemann and Solomons, 1962). In contrast, lactulose, a disaccharide of molecular radius $5 \AA$, is likely to be too large to penetrate these

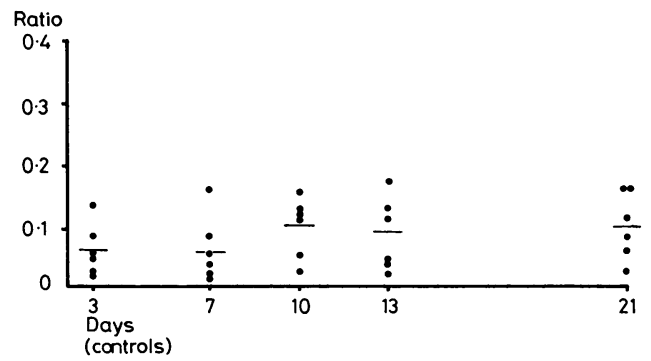

Fig. 6 Gavage experiments: lactulose/mannitol ratios in eight hour urine collections in control rats. 
pores, and this is reflected in the much lower quantities recovered. Mannitol is subject to a certain degree of hepatic metabolism, although this seems to be relatively constant even in liver disease states (Nasrallah and Iber, 1969). There is no reason to suppose that hepatic function is changed in $N$. brasiliensis infections; it would be difficult to explain a decreased urinary recovery on this basis unless one postulated enhanced hepatic metabolism. Small amounts of mannitol may be produced endogenously (Ingram et al., 1971). By using a relatively large oral dose, we are able to incorporate a 200 -fold dilution of urine samples into the assay method, which virtually eliminates this effect. In addition, for all samples the spectrophotometer was zeroed against a corresponding blank urine obtained from the rat in the ungavaged state. In practice, these blank urines almost always contained undetectable quantities of mannitol. Lactulose is not hydrolysed by the small intestine (Dahlquist and Gryboski, 1965), and can be recovered almost totally in the urine after intravenous injection (Menzies, 1974). As some crosscontamination between urine and faeces can occur in metabolism cages, it could be argued that the results in the gavage experiment might reflect increased faecal contamination occurring as a result of the looseness of stool associated with the infection. However, in many cases, no faeces collected during the eight-hour period, and homogenates of faeces that were produced in this time, even by infected animals, contained undectable amounts of either substance. Serial gavaging with hypertonic solutions (using $5 \mathrm{ml}$ volumes on a daily basis) has been shown to decrease intestinal disaccharidase function, without altering intestinal structure (Pergolizzi et al., 1977). The control group showed no significant change in ratio as a result of serial gavages, showing that the effects are not due to this factor.

Symons et al. (1971) stressed that results for absorption in closed loop experiments should be expressed in terms of weight of tissue rather than intestinal length in $N$. brasiliensis infection, because of the intestinal dilatation that occurs. As there is a significant increase in tissue mass of non-mucosal elements-for example, smooth muscle (Fig. 1) during the infection, this would seem to lead to greater inaccuracy: what is likely to be relevant is the change in surface area. There has been said to be a very slight increase in epithelial cell numbers due to the intestinal dilatation (Symons and Fairbairn, 1960), notwithstanding the decreased villous heights. This does not take into account changes in microvilli, which are quantitatively the most important factor determining intestinal absorption area. One would expect that, as in coeliac disease, the microvilli would be both fewer and stunted as a result of the increased cell turnover and lack of maturation of epithelial cells, and this effect has been described (Symons et al., 1971). In any case, any correction factor required to describe the result in terms of tissue weight would apply to both molecules, and the increase in ratio would be unaffected.

How do we account for the contrasting changes in permeability to the two molecules? Mannitol, as discussed previously, is probably just small enough to penetrate the transcellular aqueous pores of the small intestine. If there is a reduction in absorptive surface area, then there will be fewer of these pores available to the mannitol molecules. Alternatively, the pores may become smaller, in the way that Fordtran et al. (1967) have suggested occurs in sprue. Increased permeability to intact proteins, which is known to occur in coeliac disease (Kivel et al., 1964), has also been demonstrated in rats infected with $N$. brasiliensis (Barth et al., 1966), and is believed to assist in the expulsion of the parasites by allowing antibodies to come into contact with them. We have now shown that the increased permeability extends to molecules of the size of disaccharides, which is yet another point of similarity between the intestinal lesion and that occurring in coeliac disease. The pores through which lactulose penetrates the intestine in normal circumstances must be larger than the 'typical' pore, and much fewer in number.

There is evidence to suggest that this second set of larger pores is represented by the so-called tight junctions between adjacent cells (Wright and Pietras, 1974). Another route by which large molecules might gain access is through the gaps left by cells shed at the extrusion zones at the tips of villi. The increased intestinal permeability to lactulose which occurs in rats infected with $N$. brasiliensis (and also in coeliac disease) could thus be accounted for in three different ways. Firstly, there could be some functional change in the tight junctions. Alternatively, there may be non-specific gaps in the mucosa related to damage by the parasite or the immune reaction. Finally, the increased cell turnover implies an increase in the number of extrusion zones, through which large molecules could pass.

Whatever the explanation, it is clear that there are striking similarities between the changes in intestinal structure and function found in human coeliac disease and those found in rats infected with Nippostrongylus brasiliensis.

These experiments tend to support the results we have shown in human coeliac disease, and confirm the value of simultaneously administering two molecules of differing behaviour as an oral test of intestinal permeability.

We are grateful to Professor D. L. Lee and the staff 
of the Agricultural Zoology Department, Leeds University, for providing the larvae, to Dr D. G. Jones and his colleagues of the Animal Studies Group, Safety of Medicines Department, ICI, Alderley Edge, for demonstrating the technique of bladder cannulation, and to $\mathrm{Dr} \mathrm{M}$. F. Dixon, Department of Pathology, for the histology. We also wish to thank the staff of the Laboratory Animal Services Department of Leeds University Medical School for their help in providing cages and space for experimentation, the Professorial Medical Unit for laboratory facilities, and Mrs C. L. Baxendale for typing this manuscript. IC is in receipt of a grant from the West Riding Research Fund.

\section{References}

Alvarado, F. (1966). D-xylose active transport in the hamster small intestine. Biochimica et Biophysica Acta, 112, 292-306.

Barth, E. E. E., Jarrett, W. F. H., and Urquhart, G. M. (1966). Studies on the mechanism of the self-cure reaction in rats infected with Nippostrongylus brasiliensis. Immunology, 10, 459-464.

Cobden, I., Dickinson, R. J., Rothwell, J., and Axon, A. T. R. (1978a). A non-invasive approach to intestinal permeability in coeliac disease. Gut, 19, A437-438.

Cobden, I., Dickinson, R. J., Rothwell, J., and Axon, A. T. R. (1978b). Intestinal permeability assessed by excretion ratios of two molecules: results in coeliac disease. British Medical Journal, 2, 1060.

Corcoran, A. C., and Page, I. H. (1947). A method for the determination of mannitol in plasma and urine. Journal of Biological Chemistry, 170, 165-171.

Croft, D. N., Loehry, C. A., and Creamer, B. (1968). Small bowel cell loss and weight loss in the coeliac syndrome. Lancet, 2, 68-70.

Dahlquist, A., and Gryboski, J. D. (1965). Inability of the small intestine to hydrolyse lactulose. Biochimica et Biophysica Acta, 110, 635-636.

Ferguson, A., and Jarrett, E. E. E. (1975). Hypersensitivity reactions in the small intestine. Gut, 16, 114-117.

Fordtran, J. S., Rector, F. C., Maynard, F. G., Soter, N., and Kinney, J. (1967). Water and solute movement in the small intestine of patients with sprue. Journal of Clinical Investigation, 46, 287-298.
Ingram, P, Applegarth, D. A., Sturrock, S., and Whyte, J. N. C. (1971). Sorbitol and mannitol in urine. Clinica Chimica Acta, 35, 523-524.

Jenkins, D. C. (1974). Nippostrongylus brasiliensis: distribution of primary worm population within the small intestine of neonatal rats. Parasitology, 68, 339-345.

Kivel, R. M., Kearns, D. H., and Liebowitz, D. (1964). Significance of antibodies to dietary proteins in the serums of patients with non-tropical sprue. New England Journal of Medicine, 271, 769-772.

Lindemann, B., and Solomons, A. K. (1962). Permeability of luminal surfaces of intestinal mucosal cells. Journal of General Physiology, 45, 801-810.

Loehry, C. A., and Creamer, B. (1969). Three-dimensional structure of rat small intestinal mucosa related to mucosal dynamics. Gut, 10, 112-120.

Menzies, I. S. (1973). Quantitative estimation of sugars in blood and urine by paper chromatography using direct densitometry. Journal of Chromatography, 81, 109-127.

Menzies, I. S. (1974). Absorption of intact oligosaccharide in health and disease. Biochemical Society Transactions, 2 , 1042-1047.

Nasrallah, S. M., and Iber, F L. (1968). Mannitol absorption and metbolism in man. Federation Proceedings, 27, 574.

Nasrallah, S. M., and Iber, F. L. (1969). Mannitol absorption and metabolism in man. American Journal of the Medical Sciences, 258, 80-88.

Pergolizzi, R., Lifshitz, F., Jeichberg, S., and Wapnir, R. A. (1977). Interaction between dietary carbohydrates and intestinal disaccharides in experimental diarrhoea. American Journal of Clinical Nutrition, 30, 482-489.

Symons, L. E. A. (1965). Kinetics of the epithelial cells and morphology of villi and crypts in the jejunum of rats infected by the nematode Nlpponstrongylus brasiliensis. Gastroenterology, 49, 158-168.

Symons, L. E. A., and Fairbairn, D. (1960). Pathology, absorption, transport and activity of digestive enzymes in rat jejunum parasitized by the nematode Nippostrongylus brasiliensis. Federation Proceedings, 21, 913-918.

Symons, L. E. A., and Fairbairn, D. (1963). Biochemical pathology of the rat jejunum parasitised by the nematode Nippostrongylus brasiliensis. Experimental Parasitology, 13, 284-304.

Symons, L. E. A., Gibbins, J. R., and Jones, W. O. (1971). Jejunal malabsorption in the rat infected by the nematode Nippostrongylus brasiliensis. International Journal for Parasitology, 1, 179-187.

Wright, E. M., and Pietras, R. J. (1974). Routes of nonelectrolyte permeation across epithelial membranes. Journal of Membrane Biology, 17, 293-312. 